Leonardo FAVILLI*

\title{
I Ropaloceri del litorale a duna della ZSC IT5160004 Padule di Bolgheri (Livorno, Toscana) (Lepidoptera)
}

\begin{abstract}
Riassunto: Nel 2015 è stata studiata la fauna a Ropaloceri del litorale a duna della ZSC IT5160004 Padule di Bolgheri (provincia di Livorno). I campionamenti sono stati effettuati due volte al mese, da aprile a ottobre, in un transetto di $1 \mathrm{~km}$ utilizzando il metodo di Pollard \& Yates (1993). Complessivamente sono stati campionati 422 esemplari attribuibili a 30 specie. Le specie dominanti sono 7 (Lasiommata megera, Leptotes pirithous, Colias crocea, Gonepteryx rhamni, Pieris brassicae, Gonepteryx cleopatra, Hipparchia statilinus, Coenonympha elbana, Anthocharis cardamines, Pieris rapae e Limenitis reducta), abbondanti 4, 7 comuni, 7 occasionali e 5 rare. Rilevante è la presenza di Gegenes nostrodamus, in diminuzione in Italia e di Coenonympha elbana, endemica della Toscana. Si tratta di una fauna povera in specie, costituita da entità euriece e che non sono esclusive dell'ambiente di duna. Nel sistema a duna indagato i Ropaloceri sono, quindi, rappresentati in modo limitato e non annoverano entità utili a caratterizzarle in maniera univoca.
\end{abstract}

\begin{abstract}
The rhopaloceran fauna of the dunes of the ZSC IT5160004 Padule di Bolgheri (province of Livorno, Tuscany, Italy). In the year 2015 it was studied the rhopaloceran fauna of the dunes of the ZSC IT5160004 Padule di Bolgheri (province of Livorno). The samples were carried out twice a month from April to October, in a $1 \mathrm{~km}$ transect using the method proposed by Pollard \& Yates (1993). A total of 422 specimens attributed to 30 species were sampled. The dominant species are 7 (Lasiommata megera, Leptotes pirithous, Colias crocea, Gonepteryx rhamni, Pieris brassicae, Gonepteryx cleopatra, Hipparchia statilinus, Coenonympha elbana, Anthocharis cardamines, Pieris rapae e Limenitis reducta), abundant 4, 7 common, 7 occasional and 5 rare. Significant is the presence of Gegenes nostrodamus, decreasing in Italy, and of Coenonympha elbana, endemic of Tuscany. It is a fauna poor in species, consisting of entities characterized by a high ecological niche and are not exclusive to the dune environment. Ropalocerans, then, are poorly represented in the dunes and they do not include species useful to the characterization of this habitat.
\end{abstract}

Key words: Rhopalocera, ZSC IT5160004 Padule di Bolgheri, checklist, dominant species, significant species for conservation value.

\section{INTRODUZIONE}

Nel recente passato centinaia di chilometri di litorali sabbiosi in tutta Italia sono stati occupati da aree residenziali, infrastrutture viarie, parcheggi, stabilimenti balneari e porti turistici che hanno determinato la quasi totale distruzione delle dune che li caratterizzavano (Onori et al., 2013; Acosta \& Ercole, 2015).

Le dune sono rilievi sabbiosi che si originano parallelamente alla linea di costa in seguito all'azione congiunta delle onde e delle correnti marine. Si tratta di ambienti estremamente dinamici, soggetti a continui apporti di umidità da parte del mare e costantemente rimodellati dall'attività dei venti e delle correnti, nei quali la mobilità e l'aridità del substrato, la salinità, l'accentuata insolazione, le alte temperature estive e la ventilazione costante costituiscono i più significativi fattori limitanti che rendono difficile la colonizzazione da parte delle specie vegetali e animali
(Audisio, 2002; Acosta \& Ercole, 2015). Per il loro elevato valore naturalistico, dovuto alla presenza di habitat di interesse conservazionistico, alcuni dei quali a priorità di conservazione (2250 Dune costiere con Juniperus spp.; 2270 Dune con foreste di Pinus pinea e/o Pinus pinaster), le dune sono protette in tutta Italia e in Europa ai sensi della Direttiva 92/43/CEE.

Litorali intatti o comunque poco alterati si sono conservati dove l'utilizzo della spiaggia si è mantenuto a livelli più bassi o dove sono stati istituite aree protette. Qui sopravvive una fauna peculiare, adattata all'ambiente di duna attraverso particolari forme di adattamento (sviluppo di attività fossorie, strategie atte a minimizzare il contatto con il substrato, modificazioni fisiologiche degli apparati escretori, adattamenti tegumentari, mimetismo, ecc.) e spesso costituita da elementi di assoluto interesse scientifico (endemiti, specie al limite dell'areale di distribuzione) e conser-

\footnotetext{
*Leonardo Favilli, Dipartimento di Scienze Fisiche della Terra e dell'Ambiente, Via Mattioli 4, 53100 Siena (SI), Italia. E-mail: leonardo.favilli@unisi.it
} 
vazionistico (specie minacciate a scala locale, nazionale, globale) (Audisio, 2002; La Greca, 2002).

Nonostante la particolarità e l'importanza dei popolamenti animali dei litorali sabbiosi italiani, le conoscenze acquisite sino a oggi sono abbastanza limitate. Uno dei gruppi meno studiati è quello dei Ropaloceri, sebbene sia tra quelli meglio rappresentati negli ecosistemi terrestri. Gli unici lavori che trattano espressamente i Ropaloceri delle dune italiane sono quelli di Balletto \& Toso (1982) e di Scalercio (2001), che prendono in esame una trentina di località dell'Italia meridionale (Sicilia, Calabria, Campania e Puglia); poche altre notizie sono reperibili nei lavori di Arrigoni et al. (1976), Terzani (1982), Camporesi \& Fiumi (1983), Fabbris (1990), Zilli et al. (2001), Dapporto et al. (2005), Nappini \& Dapporto (2009) e Piazzini et al. (2012), che ricordano la presenza di specie osservate nelle aree costiere romagnole, toscane e laziali, o sono disperse in contributi che hanno per oggetto comprensori ben più vasti (per una rassegna bibliografica al riguardo cf. Balletto et al., 2007).

Scopo di questo lavoro è contribuire a migliorare la conoscenza sulla fauna a Ropaloceri dei litorali sabbiosi italiani accertando le specie presenti in un'area della costa tirrenica toscana e individuando le entità più rappresentative dal punto di vista faunistico e conservazionistico.

\section{Materiali e Metodi}

Area di studio. Per realizzare questo studio è stata individuata una Zona Speciale di Conservazione (ZSC) della Toscana, la ZSC IT5160004 Padule di Bolgheri (Castagneto Carducci, Livorno), caratterizzata da litorali sabbiosi ancora abbastanza integri (Fig. 1).

Nel litorale di questa ZSC è ancora ben rappresentata la vegetazione psammofila caratteristica delle spiagge sabbiose, distribuita secondo una zonazione ben definita, procedendo dal mare verso l'interno. Nelle zone immediatamente successive alla sabbia nuda, soggetta al moto ondoso, sono presenti poche piante pioniere, come il ravastrello marittimo (Cakile marittima), la salsola erba cali (Kali tragus) e l'euforbia marittima (Euphorbia paralias). In corrispondenza delle dune embrionali, solo sporadicamente invase dal mare, si trovano, invece, entità un po' più esigenti nella scelta del substrato come lo sporobolo delle spiagge (Sporobolus virginicus) mentre ancora più all'interno, dove le dune sono in fase di consolidamento, entità che con i loro apparati radicali tendono a favorire questo processo tra le quali la calcatreppola marittima (Eryngium maritimum). I retroduna consolidati e, quindi, più stabili, sono colonizzati dal giglio marino comune (Pancratium maritimum), dalla crucianella marittima (Crucianella marittima) e dal perpetuino profumato (Helichrysum stoechas); ad essi fanno seguito specie come i ginepri (Juniperus spp.), il mirto (Pistacia lentiscus) e il lentisco (Myrtus communis) che vegetano al limite interno del litorale sabbioso e segnano il passaggio dai sistemi dunosi veri e propri alle macchie e alle foreste originariamente a leccio (Quercus ilex), oggi quasi del tutto sostituite da impianti artificiali a pini (Pinus pinaster e Pinus pinea) (De Dominicis, 1993; Pignatti, 2002; Ciccarelli et al., 2015).

Il clima è di tipo mediterraneo, con temperatura e piovosità medie annue rispettivamente di $16^{\circ} \mathrm{C}$ e 775 mm (Barazzuoli et al., 1993; Regione Toscana, 2017).

L'area è nel complesso poco antropizzata per la mancanza di insediamenti residenziali, di centri produttivi e di infrastrutture viarie e per la ridotta presenza antropica nel litorale durante nei mesi estivi. Ciò è dovuto al fatto che la superficie della ZSC coincide con quella dell'Oasi WWF Rifugio Faunistico Padule di Bolgheri che da circa sessanta anni garantisce una gestione a fini conservazionistici del territorio e ne ha impedito sia l'eccessivo sfruttamento agricolo sia l'urbanizzazione.

Campionamento. Per i campionamenti è stato usato il metodo del "transect en zigzag" proposto da Ouin et al. (2000) a modifica del transetto proposto da Pollard \& Yates (1993). Nell'area di studio è stato individuato un transetto di $1 \mathrm{~km}$ di lunghezza con percorso sinuoso battigia-limite interno del litorale, in modo da visitare tutte le tipologie vegetazionali presenti ed accertare il maggior numero di specie di Ropaloceri possibile. Ogni transetto è stato percorso alla velocità costante (per quanto possibile) annotando le specie e il numero di esemplari osservati in volo o posati all'interno di un box immaginario $3 \mathrm{~m}$ da ciascun lato e $6 \mathrm{~m}$ davanti e sopra all'operatore.

I campionamenti sono stati effettuati due volte al mese, da aprile a ottobre $2015(04.04,19.04,03.05$, $17.05,06.06,21.06,05.07,19.07,02.08,16.08,05.09$, $19.09,03.10,17.10)$, eseguendo i rilievi nelle ore di massima attività degli adulti (tra le 10:00 e le 16:00 ora solare), con tempo sereno e intensità del vento $\leq 5$ della scala Beaufort (Pollard \& Yates, 1993). 
I Ropaloceri del litorale a duna della ZSC IT5160004 Padule di Bolgheri (Livorno, Toscana)

MINISTERO DELL'AMBIENTE
EDELLATUTELA DEL TERRTIORIO E DELL MARE

Regione: Toscana

Codice sito: IT5160004

Denominazione: Padule di Bolgheri

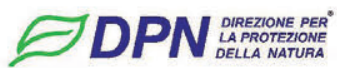

Superficie (ha): 577

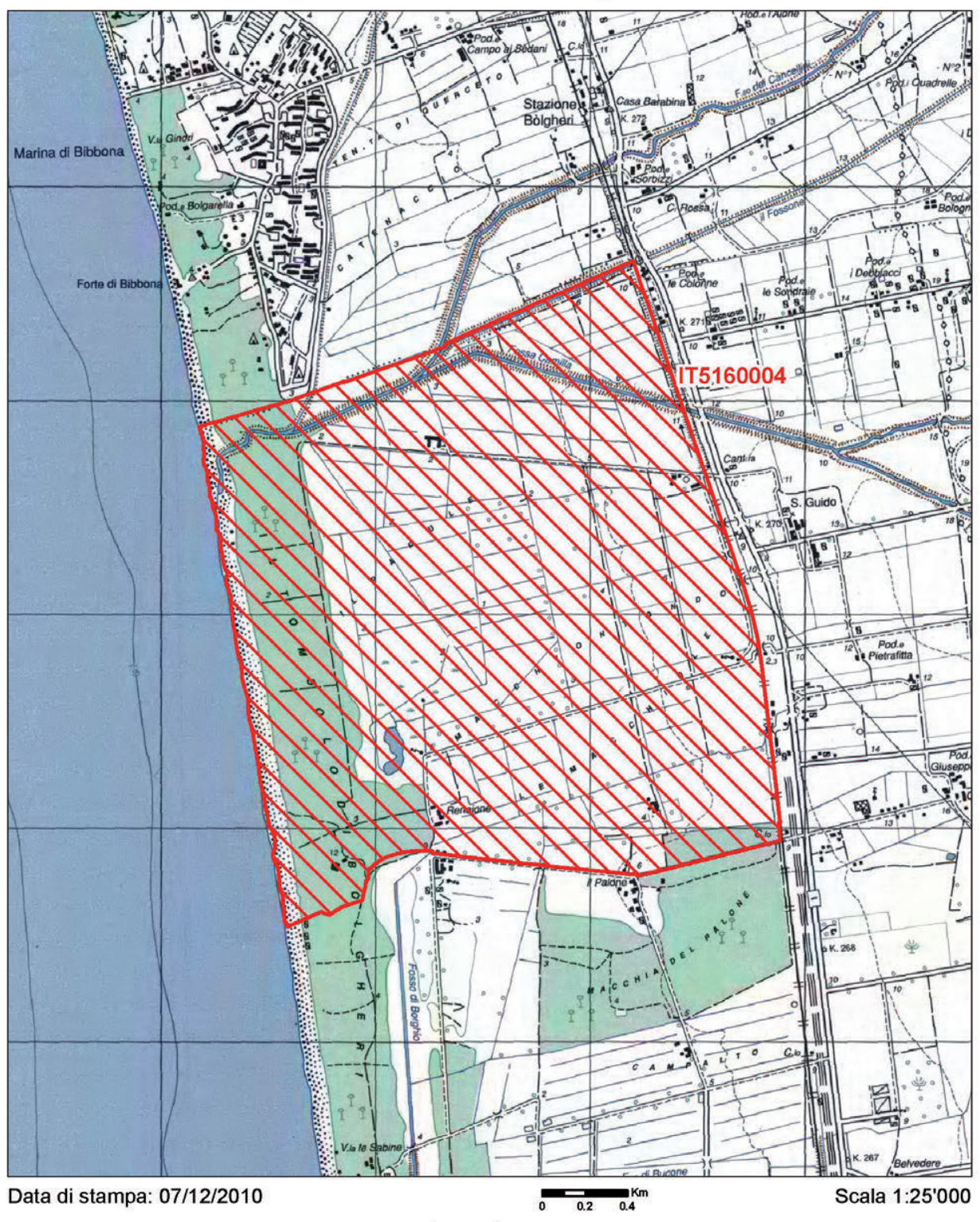

Legenda

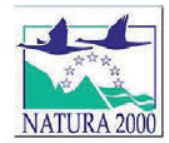

$\Delta \nabla$ sito IT5160004
$\square$ altri siti

Base cartografica: IGM 1:25'000

Fig. 1 - Area di studio. Ripreso da Ministero dell'Ambiente e della Tutela del Territorio e del Mare, 2017. Disponibile da: ftp://ftp.minambiente.it/PNM/Natura2000/TrasmissioneCE_dicembre2017/schede_mappe/Toscana/ZSC_mappe/IT5160004_A4-vert.jpg 
Le farfalle incontrate all'interno di ciascun transetto, escluse quelle determinabili direttamente a vista, sono state catturate utilizzando un retino entomologico, determinate ricorrendo al metodo proposto da Lafranchis (2004) (che permette di esaminare gli stessi sul campo ricorrendo a una lente di ingrandimento, senza doverli sacrificare per portarli in laboratorio per la determinazione) e immediatamente rilasciate.

Per la tassonomia e la nomenclatura si è fatto riferimento a Balletto et al. (2014), per le preferenze ecologiche delle singole specie a Balletto \& Kudrna (1985) e per le classi di frequenza a Parsons (1991).

\section{RISULTATI}

Complessivamente sono stati campionati 422 esemplari attribuibili a 30 specie così suddivise: 2 appartengono agli Hesperidae, 2 ai Papilionidae, 10 ai Pieridae, 6 ai Lycaenidae e 10 ai Nymphalidae (Tab. 1).

Dal punto di vista ecologico prevalgono le specie subnemorali $(66,7 \%)$, le eliofile $(56,7 \%)$, le mesoterme $(43,3 \%)$, le mesoigre $(66,7 \%)$ e le sedentarie o poco mobili (53,3\%) (Tab. 2).

Le specie dominanti sono 7, L. megera, L. pirithous, C. crocea, G. rhamni, P. brassicae, G. cleopatra e $H$. statilinus con le prime 3 che hanno totalizzato i valori di classe di frequenza di gran lunga più elevati; delle rimanenti 24 specie, $4, A$. cardamines, $C$. elbana, $P$. rapae e $L$. reducta sono abbondanti e 7, V. atalanta, G. nostrodamus, P. machaon, L. phlaeas, P. icarus, V. cardui e C. pamphilus, comuni, mentre le restanti 12 risultano occasionali o rare (Tab. 1).

Le entità più interessanti dal punto di vista faunistico e conservazionistico sono G. nostrodamus e C. elbana.

Il primo è un esperide a corotipo centroasiaticomediterraneo poco comune e considerato in declino in Italia dove è diffuso dalla Liguria alla Sicilia (manca in Sardegna) (Balletto et al., 2007, 2016).

C. elbana è un ninfalide a corotipo appenninico, con areale limitato all'Arcipelago Toscano (Capraia, Elba, Giannutri) e ad alcune stazioni della Toscana continentale in provincia di Livorno e Grosseto (Casini, 2017). È minacciata dagli incendi, che periodicamente colpiscono incolti e macchia mediterranea, dal pascolo degli ungulati selvatici e domestici, che danneggia la vegetazione spontanea, e dall'urbanizzazione delle aree costiere e recentemente si è estinta nell'Isola del Giglio (Fabiano et al., 2001; Balletto et al., 2016).

\section{DiscusSIONE E CONCLUSIONI}

Con 30 specie la ropalocerofauna delle dune della ZSC Padule di Bolgheri risulta povera ma più ricca di quella delle coste sabbiose della Campania, della Calabria, della Puglia e della Sicilia studiate da Balletto \& Toso (1982) e da Scalercio (2001) (Tab. 3).

Quasi tutte le entità rinvenute frequentano un'ampia varietà di situazioni ambientali al di fuori dei litorali sabbiosi e sono largamente diffuse in Italia e in Toscana. La maggior parte di esse sono per lo più legate ad ambienti soleggiati, caldi e asciutti (prevalenza delle specie eliofile, mesoterme e mesoigre), come era logico attendersi, sono sedentarie o scarsamente mobili e non presentano problemi di conservazione, con esclusione di G. nostrodamus e C. elbana.

Le specie dominanti sono risultate 7: L. megera, L. pirithous, C. crocea, G. rhamni, P. brassicae, G. cleopatra e H. statilinus. Nelle dune della Sicilia Balletto \& Toso (1982) hanno rilevato come più frequenti 10 specie, G. nostrodamus, Carcharodus alceae (Esper, 1780), P. edusa, Gegenes pumilio (Hoffmannsegg, 1804), P. machaon, P. rapae, C. crocea, $L$. phlaeas, V. cardui e $P$. cecilia e nelle dune della Campania, Calabria e Puglia 11 specie, $P$. edusa, P. cecilia, G. nostrodamus, I. podalirius, P. rapae, C. crocea, $L$. pirithous, A. agestis, Melitaea phoebe (Denis \& Schiffermüller, 1775), H. statilinus e C. pamphilus), mentre Scalercio (2001) 5 specie in quelle della Calabria, $P$. edusa, P. rapae, E. ausonia, H. aristaeus e P. icarus.

Le entità in comune tra i litorali dell'Italia meridionale, nel loro insieme, e della ZSC Padule di Bolgheri sono 23,14 quelle trovate esclusivamente nel sud Italia e 7 quelle presenti soltanto nelle dune di Bolgheri.

Degna di nota è l'assenza di G. pumilio e di $P$. cecilia, al contrario ben rappresentate nei litorali del Meridione. Il primo è una specie mediterranea, in Italia diffusa per lo più lungo le dune costiere e in Toscana ad oggi nota con sicurezza per un numero limitato di località dell'Elba, della fascia costiera e dell'entroterra (Bartolini, 1999; Balletto et al., 2007, 2016). La scarsa presenza di questa specie in Toscana potrebbe spiegarne l'assenza dai sistemi a duna campionati. Più difficile è motivare l'assenza di $P$. cecilia; si tratta, infatti, di una specie molto comune e ampiamente diffusa in Italia e in Toscana che frequenta le radure di boschi termofili mediterranei e che predilige i siti caldi e assolati e i terreni asciutti, anche a poca distanza dal mare. Tuttavia, in oltre un ventennio di 
ricerche nel centro-sud della regione la presenza di $P$. cecilia è stata accertata più volte nelle selve costiere $\mathrm{e}$ nelle zone a macchia mediterranea alternate a pratipascoli limitrofi a sistemi a duna, ma mai in quest'ultima tipologia ambientale (L. Favilli, ined.). Si tratta di una specie polifaga su diverse specie appartenenti alla famiglia Graminaceae (Brachypodium sylvaticum, Dactylis glomerata, Agrotis capillaris, etc.), per cui è improbabile che la pianta nutrice rappresenti il fattore limitante.

Anche nella duna toscana indagata, quindi, come in quelle dell'Italia meridionale e della Sicilia (Balletto \& Toso, 1982; Scalercio, 2001), i Ropaloceri sono rappresentati in modo limitato sia in numero di specie, sia in esemplari e non annoverano entità utili a caratterizzarla in modo univoco, al contrario di

Tab. 1. Catalogo dei Ropaloceri del litorale sabbioso della ZSC Padule di Bolgheri. Classi di frequenza secondo Parsons (1991): D: dominante ( $\geq 21$ esemplari); A: abbondante (da 11 a 20 esemplari); C: comune (da 6 a 10 esemplari); O: occasionale (da 2 a 5 esemplari); R: rara (1 esemplare).

\begin{tabular}{|c|c|c|}
\hline Specie & $N^{\circ}$ exx. & Classe di frequenza \\
\hline Lasiommata megera (Linné, 1758) & 58 & $\mathrm{D}$ \\
\hline Leptotes pirithous (Linné, 1767) & 53 & $\mathrm{D}$ \\
\hline Colias crocea (Geoffroy, 1785) & 47 & $\mathrm{D}$ \\
\hline Gonepteryx rhamni (Linné, 1758) & 34 & $\mathrm{D}$ \\
\hline Pieris brassicae (Linné, 1758) & 31 & $\mathrm{D}$ \\
\hline Gonepteryx cleopatra (Linné, 1767) & 28 & $\mathrm{D}$ \\
\hline Hipparchia statilinus (Hufnagel, 1766) & 21 & $\mathrm{D}$ \\
\hline Anthocharis cardamines (Linné, 1758) & 20 & A \\
\hline Coenonympha elbana Staudinger, 1901 & 17 & A \\
\hline Pieris rapae (Linné, 1758) & 16 & A \\
\hline Limenitis reducta Staudinger, 1901 & 15 & A \\
\hline Vanessa atalanta (Linné, 1758) & 9 & $\mathrm{C}$ \\
\hline Gegenes nostrodamus (Fabricius, 1793) & 8 & $\mathrm{C}$ \\
\hline Papilio machaon Linné, 1758 & 8 & $\mathrm{C}$ \\
\hline Lycaena phlaeas (Linné, 1761) & 8 & $\mathrm{C}$ \\
\hline Polyommatus icarus (Rottemburg, 1775) & 7 & $\mathrm{C}$ \\
\hline Vanessa cardui (Linné, 1758) & 7 & $\mathrm{C}$ \\
\hline Coenonympha pamphilus (Linné, 1758) & 6 & $\mathrm{C}$ \\
\hline Pieris napi (Linné, 1758) & 5 & $\mathrm{O}$ \\
\hline Pieris edusa (Fabricius, 1777) & 4 & $\mathrm{O}$ \\
\hline Euchloe ausonia (Hübner, [1804]) & 4 & $\mathrm{O}$ \\
\hline Maniola jurtina (Linné, 1758) & 4 & $\mathrm{O}$ \\
\hline Pararge aegeria (Linné, 1758) & 3 & $\mathrm{O}$ \\
\hline Ocholdes sylvanus (Esper, [1777]) & 2 & $\mathrm{O}$ \\
\hline Iphiclides podalirius (Linné, 1758) & 2 & $\mathrm{O}$ \\
\hline Pieris mannii (Mayer, 1851) & 1 & $\mathrm{R}$ \\
\hline Lampides boeticus (Linné, 1767) & 1 & $\mathrm{R}$ \\
\hline Aricia agestis ([Denis \& Schiffermuller], 1775) & 1 & $\mathrm{R}$ \\
\hline Melitaea didyma (Esper, [1778]) & 1 & $\mathrm{R}$ \\
\hline Charaxes jasius (Linné, 1767) & 1 & $\mathrm{R}$ \\
\hline$N^{\circ}$ totale exx. & 422 & \\
\hline $\mathbf{N}^{\circ}$ specie & 30 & \\
\hline
\end{tabular}


quanto si verifica per gli Eteroceri o per altri gruppi di invertebrati come i Gasteropodi, gli Ortotteri e i Coleotteri, con entità quali Brithys crini (Fabricius, 1775), Xerosecta contermina (L. Pfeiffer, 1848), Sphingonotus personatus Zanon, 1926, Eurynebria complanata (Linné, 1767) e Pimelia bipunctata Fabricius, 1781 che si ritrovano unicamente in questo habitat (Giusti \& Castagnolo, 1982; Audisio, 2002).

Una parziale eccezione a ciò è rappresentata da L. megera e da L. pirithous, che seppure non esclusive delle dune, vi hanno fatto registrare un'alta frequenza, di gran lunga superiore a quella ottenuta dalle restanti specie. La prima è caratteristica dei prati xerici da mediterranei a montani; presenta da tre a quattro generazioni annuali, con periodo di volo esteso da aprile a ottobre ed è abbondantemente diffusa in Italia. La seconda frequenta i margini dei boschi termofili mediterranei e montani inferiori; presenta tre generazioni annuali tra marzo e novembre ed in Italia è più abbondante lungo i litorali (Balletto et al., 2007, 2016; Villa et al., 2009).
La marcata povertà faunistica della duna indagata, come suggerito per le dune italiane in genere da Audisio (2002), potrebbe essere dovuta al fatto che in questo ambiente le piante (utilizzabili per l'alimentazione dalle larve) e i fiori (utilizzabili per l'alimentazione dagli adulti) sono poco rappresentati a causa della salinità, dell'aridità e dell'incoerenza del substrato, delle elevate temperature estive, della forte escursione termica fra il giorno e la notte e della ventilazione costante, fattori che non favoriscono l'insediamento dei Ropaloceri.

La ropalocerofauna esaminata, più che da specie insediate stabilmente, sembra essere costituita da entità che giungono dalle retrostanti formazioni a macchia e boscate a pini (Pinus spp.) e a leccio (Quercus ilex), dove più elevata è la presenza di piante con fiore e, quindi, di fonti di nettare e nutrici dei bruchi e, in misura minore, da entità con tendenze migratorie (come P. brassicae, V. atalanta e V. cardui) che visitano i litorali durante i loro spostamenti.

Nei prossimi anni sarebbe interessante esten-

Tab. 2. Preferenza ecologiche dei Ropaloceri del litorale sabbioso della ZSC Padule di Bolgheri (preferenze ecologiche secondo Balletto \& Kudrna, 1985).

\section{$\mathrm{N}^{\circ}$ di specie}

\section{Habitat}

Specie subnemorali 20

Specie di formazioni erbacee aperte $\quad 7$

Specie nemorali 2

Specie di pietraie e mecereti 1

\begin{tabular}{lc}
\hline Luminosità & \\
Specie eliofile & 16 \\
Specie sciafile & 14 \\
\hline Temperatura & \\
Specie mesoterme & 13 \\
Specie macroterme & 12 \\
Specie euriterme & 5
\end{tabular}

\section{Umidità del terreno}

$\begin{array}{ll}\text { Specie mesoigre } & 20\end{array}$

$\begin{array}{ll}\text { Specie xerofile } & 7\end{array}$

Specie euriigre $\quad 3$

\section{Vagilità}

$\begin{array}{ll}\text { Specie sedentarie o poco mobili } & 16\end{array}$

$\begin{array}{ll}\text { Specie migratrici o parzialmente migratrici } & 14\end{array}$ 
Tab. 3. Checklist dei Ropaloceri del litorale sabbioso della ZSC Padule di Bolgheri, delle dune della Campania, della Calabria, della Puglia e della Sicilia (dati ripresi da Balletto \& Toso, 1982 e Scalercio, 2001).

\begin{tabular}{cccccc}
\hline Specie & $\begin{array}{c}\text { Dune ZSC } \\
\text { Padule di } \\
\text { Bolgheri }\end{array}$ & $\begin{array}{c}\text { Dune della } \\
\text { Campania }\end{array}$ & $\begin{array}{c}\text { Dune della } \\
\text { Calabria }\end{array}$ & $\begin{array}{c}\text { Dune della } \\
\text { Puglia }\end{array}$ & $\begin{array}{c}\text { Dune della } \\
\text { Sicilia }\end{array}$
\end{tabular}

Carcharodus alceae (Esper, [1780])

Pyrgus malvoides (Elwes \& Edwards, 1897)

Thymelicus acteon (Rottemburg, 1775)

Ocholdes sylvanus (Esper, [1777])

Gegenes umilio (Hoffmannsegg, 1804)

Gegenes nostrodamus (Fabricius, 1793)

Iphiclides podalirius (Linné, 1758)

Papilio machaon Linné, 1758

Leptidea sinapis (Linné, 1758)

Anthocharis cardamines (Linné, 1758)

Euchloe ausonia (Hübner, [1804])

Pieris brassicae (Linné, 1758)

Pieris mannii (Mayer, 1851)

Pieris rapae (Linné, 1758)

Pieris napi (Linné, 1758)

Pieris edusa (Fabricius, 1777)

Colias crocea (Geoffroy, 1785)

Gonepteryx rhamni (Linné, 1758)

Gonepteryx cleopatra (Linné, 1767)

Lycaena phlaeas (Linné, 1761)

Lycaena tityrus (Poda, 1761)

Lycaena thersamon (Esper, [1784])

Lampides boeticus (Linné, 1767)

Leptotes pirithous (Linné, 1767)

Aricia agestis ([Denis \& Schiffermuller], 1775)

Polyommatus icarus (Rottemburg, 1775)

Issoria lathonia (Linné, 1758)

Vanessa atalanta (Linné, 1758)

Vanessa cardui (Linné, 1758)

Polygonia egea (Cramer, [1775])

Melitaea phoebe ([Denis \& Schiffermüller], 1775)

Melitaea didyma (Esper, 1778)

Limenitis reducta Staudinger, 1901

Charaxes jasius (Linné, 1767)

Pararge aegeria (Linné, 1758)

Lasiommata megera (Linné, 1758)

Coenonympha elbana Staudinger, 1901

Coenonympha pamphilus (Linné, 1758)

Pyronia cecilia (Vallantin, 1894)

Maniola jurtina (Linné, 1758)

Satyrus ferula (Fabricius, 1793)

Hipparchia aristaeus (Bonelli, 1826)

Hipparchia statilinus (Hufnagel, 1766)

Danaus chrysippus (Linné, 1758)

$\mathrm{N}^{\circ}$ specie

Boli


dere le indagini sui popolamenti a Ropaloceri di altre aree costiere toscane e italiane; così facendo si renderebbero disponibili maggiori informazioni per valutare in modo più completo le caratteristiche della ropalo- cerofauna dei litorali sabbiosi e per proteggere in modo adeguato gli habitat che vi sono rappresentati, la cui salvaguardia è un obiettivo prioritario secondo la Direttiva 92/43/CEE (Direttiva Habitat).

\section{BIBLIOGRAFIA}

Acosta A.T.R., ERcole S. (a cura di), 2015 - Gli habitat delle coste sabbiose italiane: ecologia e problematiche di conservazione. Ispra Serie Rapporti, 215: vii + 114 pp.

Arrigoni P.V., Gellini R., Innamorati M., Lenzi M., Grillini C., Piussi P., Sartori G., Lovari S., Renzoni A., Sanesi G., 1976 - Relazione al Consorzio per l'istituzione del "Parco della Maremma". Informatore botanico italiano, 8: 283-324.

Audisio P., 2002 - Litorali sabbiosi e organismi animali, In: RuFFo S. (a cura di), Dune e spiaggie sabbiose. Ambienti fra terra e mare. Quaderni Habitat, 4. Dune e spiagge sabbiose. Ministero dell'Ambiente e della Tutela del Territorio, Museo Friulano di Storia Naturale, Udine: 63-117.

Balletto E., Bonelli B., Barbero F., Casacci L.P., Sbordoni V., Dapporto L., Scalercio S., Zilli A., Battistoni A., Teofili C., RondinINI C., 2016 - Lista Rossa delle farfalle italiane. Ropaloceri. Comitato italiano IUCN e Ministero dell'ambiente e della tutela del territorio e del mare, Roma, $47 \mathrm{pp}$.

Balletto E., Bonelli S., Cassulo L., 2007 - Lepidoptera. In: Ruffo S., Stoch F. (a cura di), Checklist and distribution of the italian fauna. Ministero dell'Ambiente e della tutela del territorio, Direzione per la protezione della natura. CD-ROM.

Balletto E., Cassulo L.A., Bonelli S., 2014 - An annotated checklist of the italian butterflies and skippers (Papilionoidea, Hesperiioidea). Zootaxa, 3583: 1-114.

Balletto E., KudRna O., 1985 - Some aspects of the conservation of butterflies in Italy, with recommendations for a future strategy (Lepidoptera Hesperiidae \& Papilionoidea). Bollettino della Società entomologica italiana, 117: 39-59.

Balletto E., Toso G., 1982 - Lepidotteri Ropaloceri dei litorali a duna dell'Italia meridionale. In: LA GRECA M. (a cura di), Quaderni sulla "Struttura delle zoocenosi terrestri". 3. Ambienti mediterranei, I. Le coste sabbiose. Consiglio Nazionale delle Ricerche. Collana del Progetto finalizzato "Promozione della qualità dell'ambiente". Pubblicazione AQ/1/177: 153-158.

Barazzuoli P., Guasparri G., SAlleolini M., 1993 - Il clima. In: Giusti F. (a cura di), La storia naturale della Toscana meridionale. Amilcare Pizzi Editore, Cinisello Balsamo (Milano): 141-171.

BARTOLINI L., 1999 - I lepidotteri ropaloceri del Padule di Fucecchio e delle Cerbaie (e altro ancora). Benedetti, Pescia (Pistoia), $176 \mathrm{pp}$.

CAMPORESI S., FiUmi G., 1983 - Ecologia delle farfalle diurne delle zone costiere di Romagna (Lepidoptera Rhopalocera). Bollettino del Museo civico di Storia naturale di Verona, 10: 79-104.

CASINI P.M., 2017 - La répartition relictuelle de Coenonympha elbana (Staudinger, 1901) en Toscane côtière et intérieure (Italie centrale) (Lepidoptera: Nymphalidae; Satyrinae). Lépidoptères Revue de Lépidoptèristes de France, 26 (67): 52-57.

Ciccarelli D., Di Bugno C., Peruzzi L., 2015 - Checklist della flora vascolare psammofila della Toscana. Atti della Società toscana di Scienze naturali residente in Pisa Memorie Serie B, 121: 37-88.

DApporto L., Magi F., Strumia F., 2005 - I macrolepidotteri dell'area boschiva e retrodunale della Riserva di San Rossore (Pisa). Frustula entomologica (Nuova Serie) 36/37: 20-45.

De Domincis V., 1993 - La vegetazione. In: Giusti F. (a cura di), La storia naturale della Toscana meridionale. Amilcare Pizzi Editore, Cinisello Balsamo (Milano): 247-341.

Fabiano F., Vignali G., Dapporto L., 2001 - Lepidotteri. In: Sforzi A., Bartolozzi L. (a cura di), Libro rosso degli Insetti della Toscana. ARSIA Regione Toscana, Firenze: 293-343.

FABBRIS S., 1990 - Le farfalle diurne del litorale grossetano (Lepidoptera, Rhopalocera). Atti del Museo civico di Storia naturale (Grosseto), 13: 37-70.

Favilli L., Piazzini S., Tellini Florenzano G., Perroud B., Manganelli G., 2012 - Nuovi dati sulla distribuzione in Toscana di alcuni lepidotteri diurni rari o poco noti (Hesperoidea, Papilionoidea). Atti della Società toscana di Scienze naturali residente in Pisa Memorie Serie B, 118: 1-8.

GiUsti F., CASTAGNOLO L., 1982 - I molluschi terrestri delle dune italiane: brevi cenni di ecologia, elenco delle specie e chiavi 
per il loro riconoscimento. In: La Greca M. (a cura di), Quaderni sulla "Struttura delle zoocenosi terrestri". 3. Ambienti mediterranei, I. Le coste sabbiose. Consiglio Nazionale delle Ricerche. Collana del Progetto finalizzato "Promozione della qualità dell'ambiente". Pubblicazione AQ/1/177: 51-102.

LA GreCA M., 2002 - Conclusioni al XXXIII Congresso della Società Italiana di Biogeografia: "La presenza dell'uomo e i problemi posti dall'uso degli ambienti naturali costieri”. Biogeographia, 33: 217-232.

LAFRANCHIS T., 2004 - Butterflies of Europe. Diatheo, Paris, 351 pp.

NAPpini S., DApporto L., 2009 - I macrolepidotteri del Parco Regionale della Maremma (Toscana) (Lepidoptera). Memorie della Società entomologica italiana, 88: 177-195.

Onori L., Battisti C., Boccalaro F. (a cura di), 2013 - SOS dune. Stato, problemi, interventi, gestione. Atti Convegno ISPRA, Roma 23 ottobre 2009, Roma, 184 pp.

Ouin A., Paillison J.M., Lonhore J., 2000 - Méthode de suivie et d'évaluation des populations et peuplements de papillons de jour. Insects, 117: 29-31.

PARsons M.J., 1991 - Butterflies of the Bulolo-Wau valley. Bishop Museum Press, Honolulu, 280 pp., 27 tavv.

Piazzini S., Spadini E., Cianchi F., Favilli L., Manganelli G., 2012 - I Lepidotteri Ropaloceri della Riserva Statale di Popolamento Animale "Lago di Burano" (Capalbio, Grosseto). Bollettino della Società entomologica italiana, 144: 99-105.

PignAtti S., 2002 - La vegetazione delle spiagge. In: Ruffo S. (a cura di), Dune e spiaggie sabbiose. Ambienti fra terra e mare. Quaderni Habitat, 4. Dune e spiagge sabbiose. Ministero dell'Ambiente e della Tutela del Territorio, Museo Friulano di Storia Naturale, Udine: 43-61.

Pollard E., Yates T., 1993 - Monitoring Butterflies for Ecology and Conservation. Chapman \& Hall, London, XIV + 244 pp.

Regione Toscana, 2017 - Servizio Idrologico Regionale. Centro funzionale regionale di monitoraggio meteo-idrologico, Firenze. www.sir.toscana.it/index.php?IDS=1\&IDSS=1. (ultimo accesso: 01.09.2017).

SCALERCIO S., 2001 - Biodiversità e sinecologia dei Lepidotteri Ropaloceri in un paesaggio mediterraneo: la Sila greca (Italia, Calabria) (Lepidoptera Hesperioidea, Papilionoidea). Rivista del Museo civico di Scienze naturali “E. Caffi” Bergamo, 20: $13-31$.

TERZANi F., 1982 - Segnalazioni Faunistiche Italiane. 17. Coenonympha elbana Staudinger (Lepidoptera Satyridae). Bollettino della Società entomologica italiana, 114: 117.

Villa R., Pellecchia M., Pesce G.B., 2009 - Farfalle d'Italia. Editrice Compositori, Bologna, 375 pp.

Zilli A., Maltzeff P., PinZari M., Raineri W., 2001 - I lepidotteri della Tenuta Presidenziale di Castelporziano (Lepidoptera). Bollettino dell'Associazione romana di Entomologia, 56: 13-48. 